\title{
Modeling of aerosol property evolution during winter haze episodes over a megacity cluster in northern China: roles of regional transport and het- erogeneous reactions of $\mathrm{SO}_{2}$
}

Huiyun Du et al.

Correspondence to: Ji Li (lijie8074@ mail.iap.ac.cn)

The copyright of individual parts of the supplement might differ from the CC BY 4.0 License. 


\section{Supplements}

2

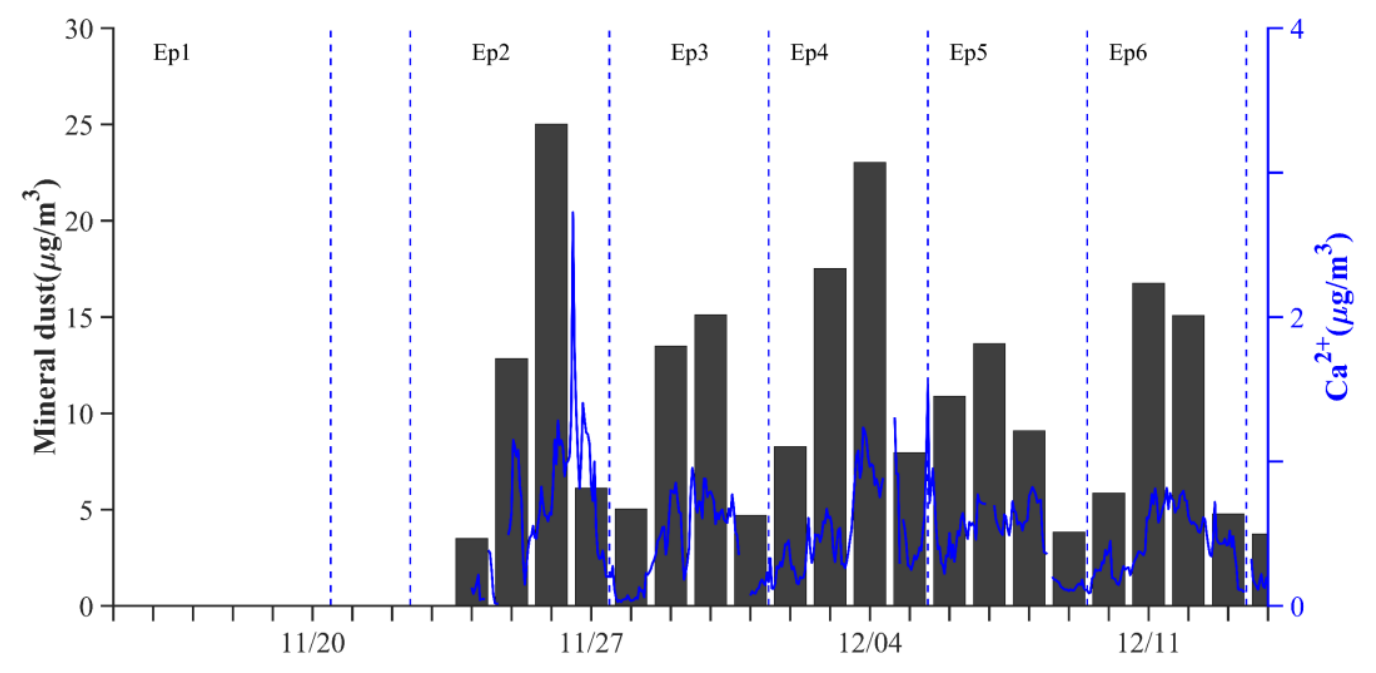

3 Figure S1. Concentration of mineral dust and $\mathrm{Ca}^{2+}$ in $\mathrm{PM}_{2.5}$ during the study period.

4 And Mineral $=2.2 \times[\mathrm{Al}]+2.49 \times[\mathrm{Si}]+1.63 \times[\mathrm{Ca}]+2.42 \times[\mathrm{Fe}]+1.94 \times[\mathrm{Ti}]$.
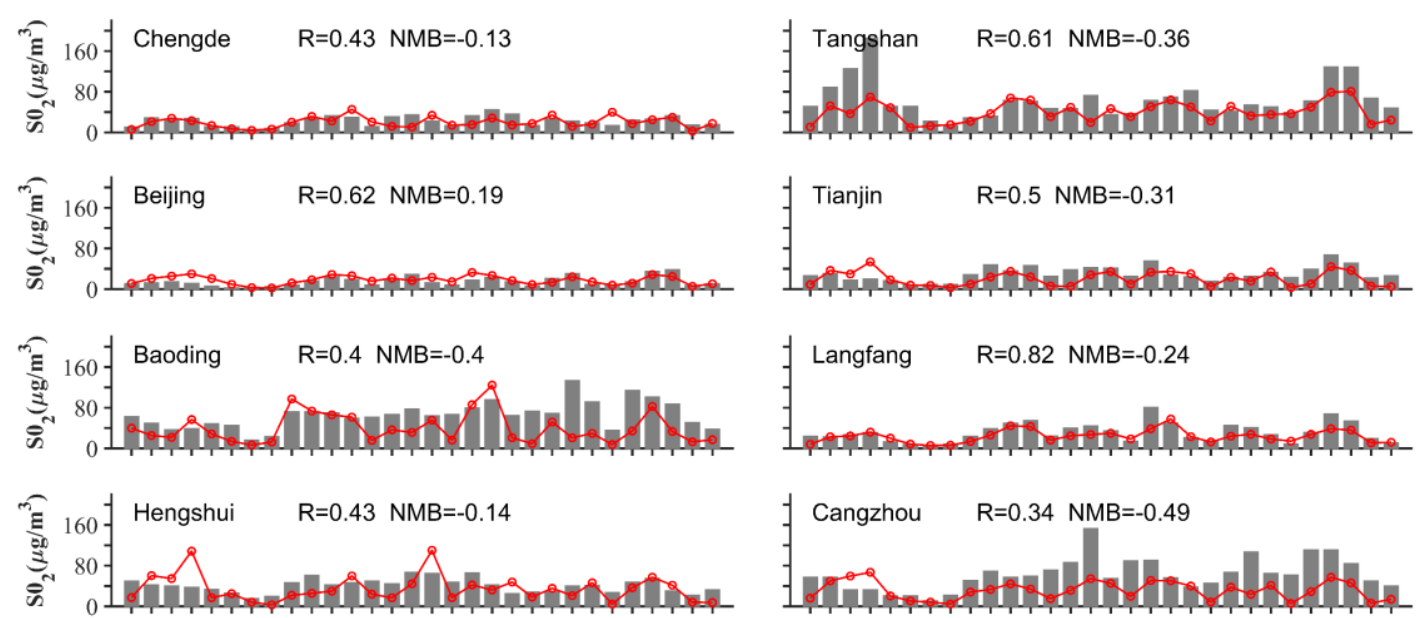

$$
(1118 \quad 11122 \quad 11126 \quad 11130 \quad 1214 \quad 1218 \quad 1212
$$

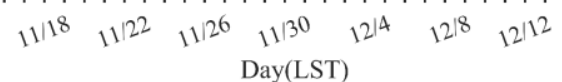
Day(LST)

$6 \quad$ Figure S2. Comparison between the simulated (red) and observed (gray bars) daily concentrations of $\mathrm{SO}_{2}$. 

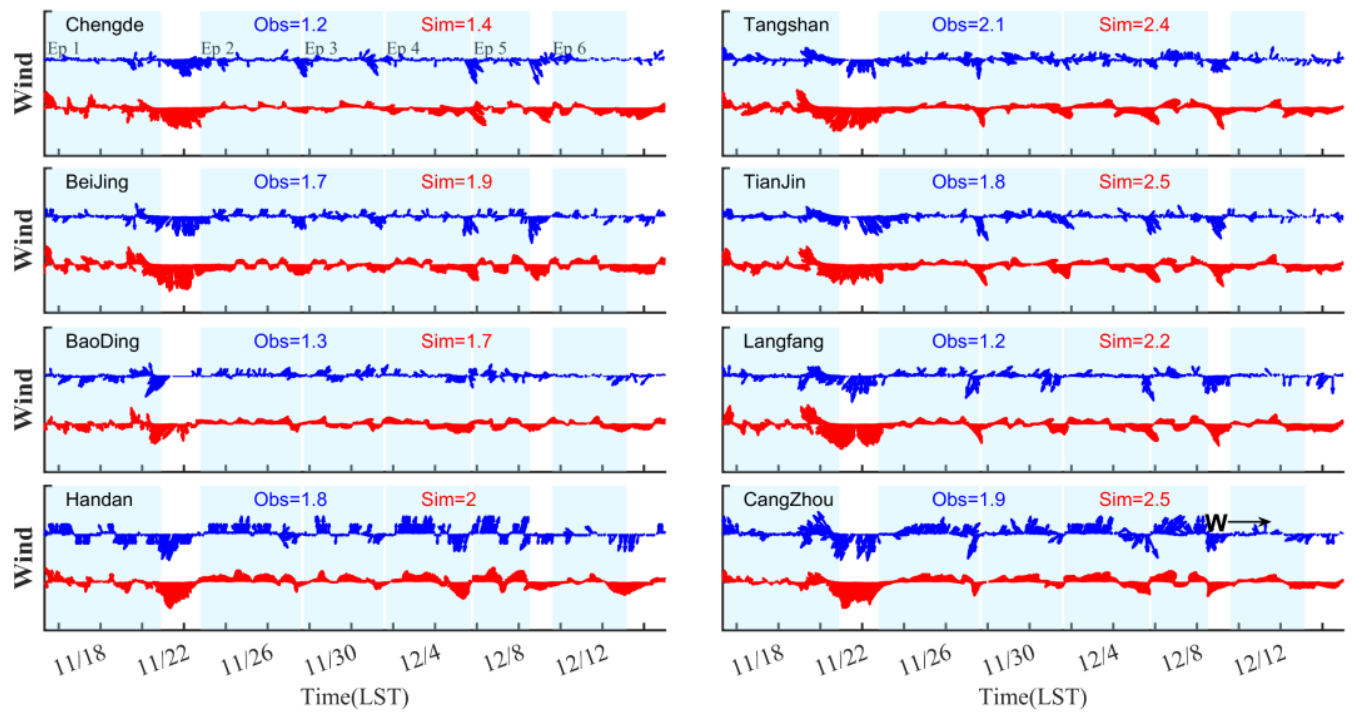

$9 \quad$ Figure S3. Comparison between simulated (red) and observed (blue) wind vector and wind speeds (data) at cities of BTH.
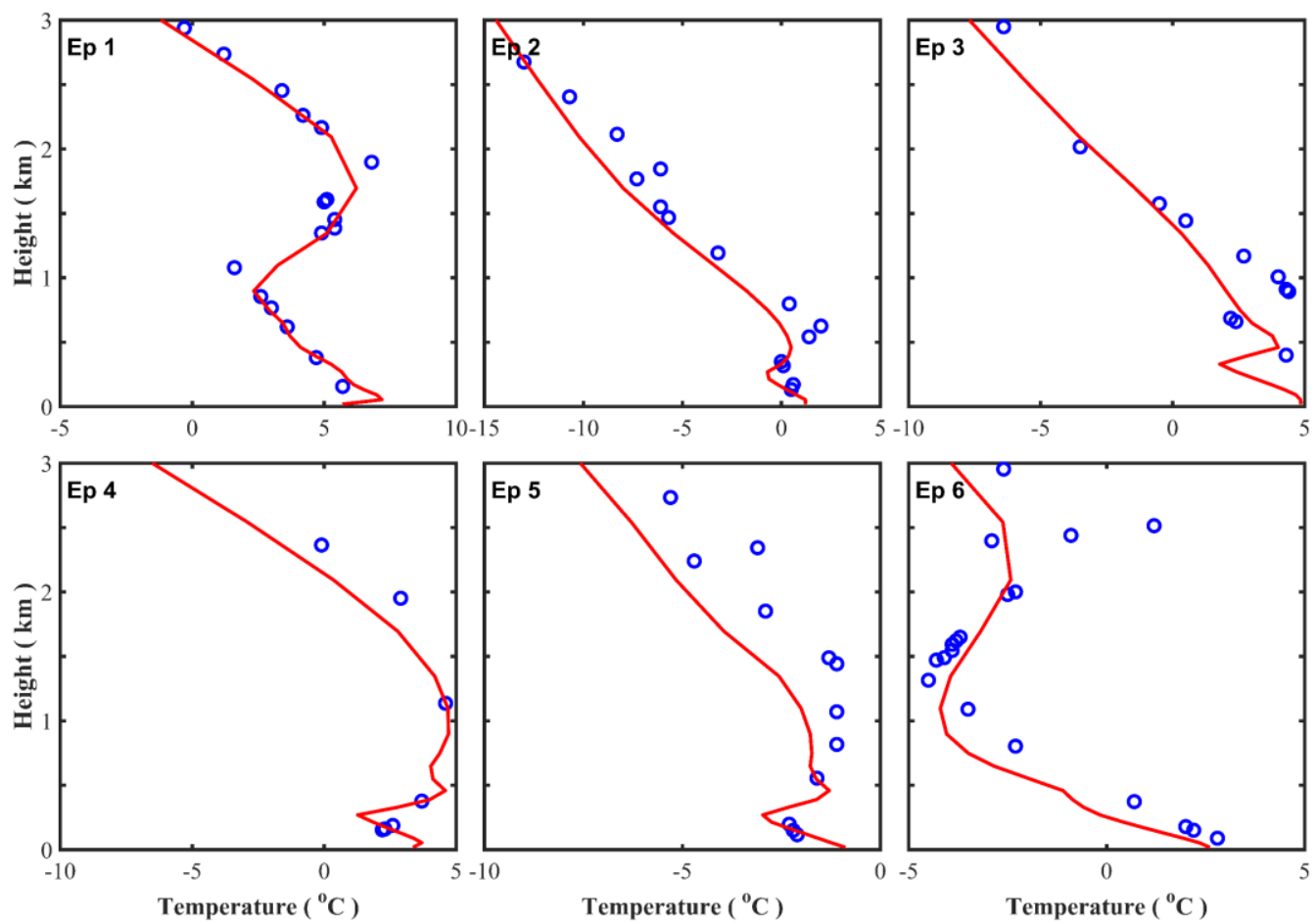

Figure S4. Comparison between simulated (red solid line) and observed (blue dot) temperature profiles during episodes in Beijing. 

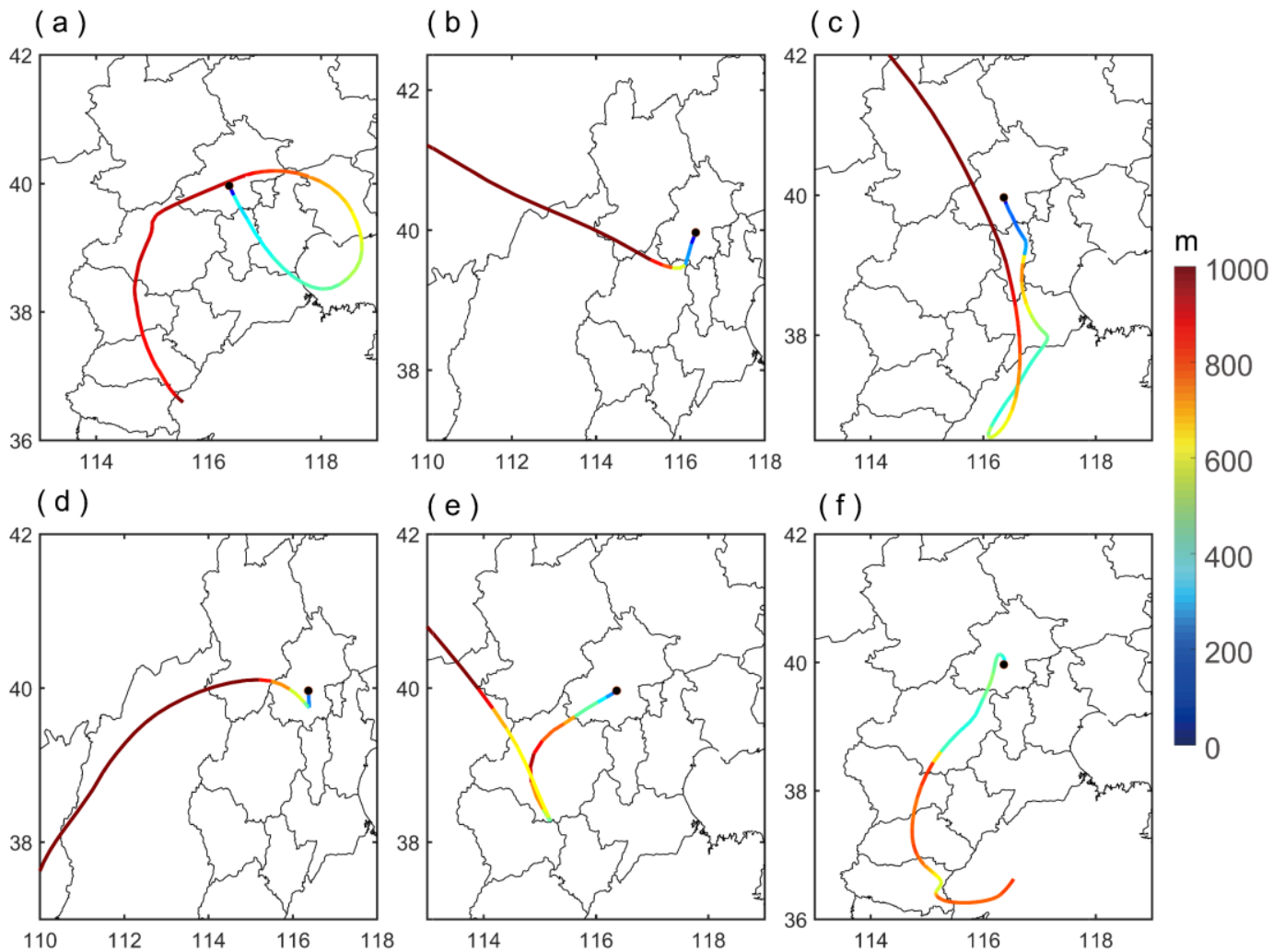

Figure S5. 72 h backward trajectories during different episodes (05:00 on November 20, 18:00 on November 26, 23:00 on November 29, 20:00 on December 03, 05:00 on December 08 and 11:00 on December 12 [LST]) at Beijing at 100m. Line color represents height of trajectories. a-f refer to Ep1-6. 


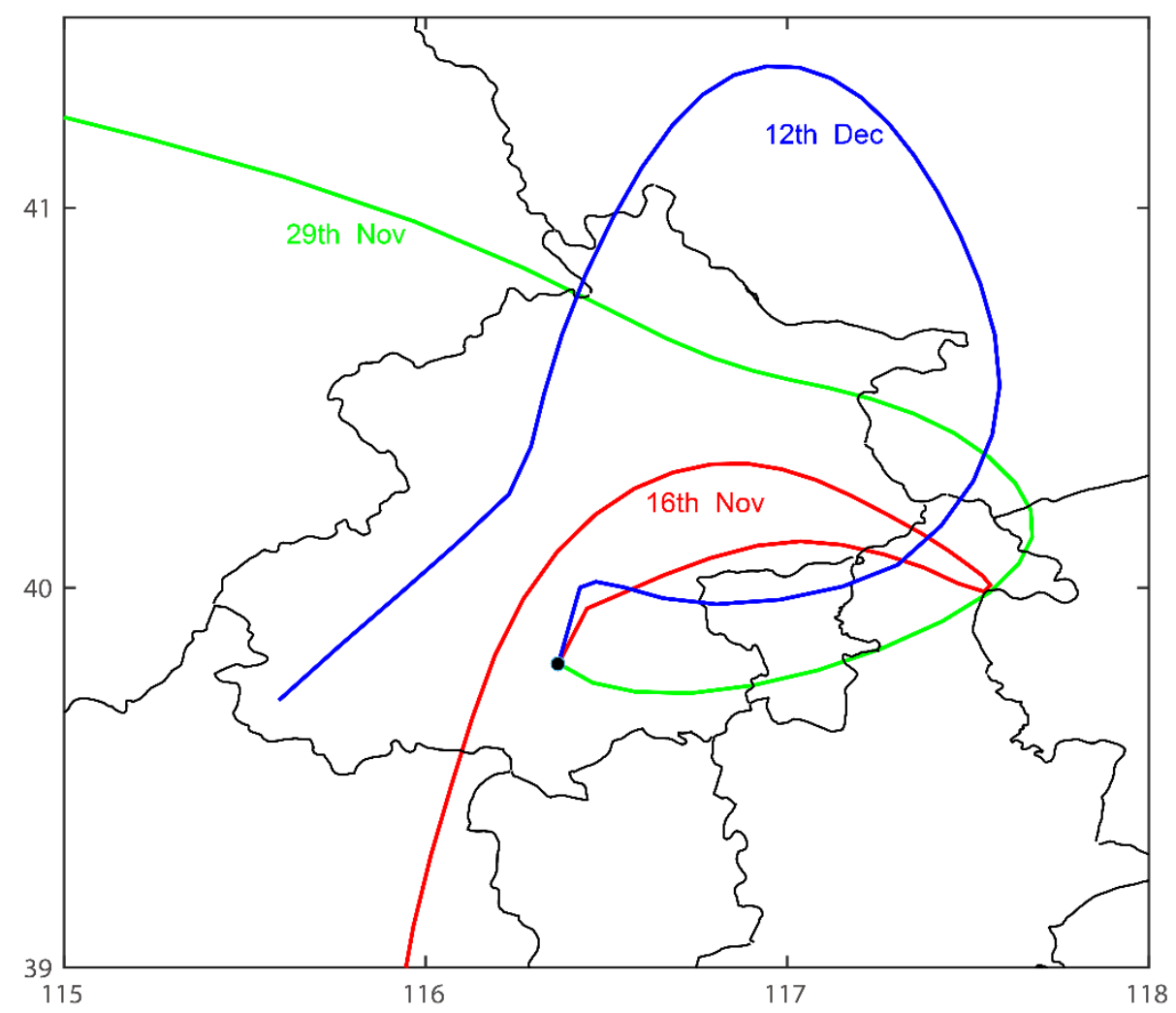

20 Figure S6. $36 \mathrm{~h}$ backward trajectories at different start time (02:00 on November 17,

21 11:00 on November 29, and 23:00 on December 12 [LST]) at Beijing.

22 Table S1. Statistics performances of meteorological simulations, including temperature 23 and relative humidity at $2 \mathrm{~m}$, and wind speed at $10 \mathrm{~m}$. Statistical parameters include 24 correlation coefficient (R), Normalized Mean Bias (NMB) and Root Mean Squared 25 Error (RMSE).

\begin{tabular}{|c|c|c|c|c|c|c|}
\hline & & Obs & Sim & NMB & $\mathrm{R}$ & RMSE \\
\hline \multirow{4}{*}{$\mathbf{T} 2(\mathrm{~K})$} & Beijing & 2.42 & 3.31 & 0.37 & 0.88 & 2.11 \\
\hline & Tianjin & 4.05 & 3.73 & -0.08 & 0.93 & 1.48 \\
\hline & Langfang & 2.13 & 3.06 & 0.44 & 0.89 & 2.13 \\
\hline & Chengde & -3.39 & -1.66 & -0.51 & 0.89 & 3.31 \\
\hline \multirow{3}{*}{ RH2(\%) } & Beijing & 53.93 & 39.53 & -0.27 & 0.69 & 21.87 \\
\hline & Tianjin & 56.95 & 47.58 & -0.16 & 0.73 & 18.09 \\
\hline & Langfang & 60.39 & 46.54 & -0.23 & 0.71 & 21.54 \\
\hline
\end{tabular}




\begin{tabular}{ccccccc}
\hline & Chengde & 61.56 & 55.96 & -0.09 & 0.47 & 20.26 \\
& Beijing & 1.68 & 1.93 & 0.15 & 0.65 & 1.30 \\
& Tianjin & 1.76 & 2.46 & 0.39 & 0.70 & 1.50 \\
& Langfang & 1.23 & 2.15 & 0.74 & 0.57 & 1.56 \\
& Chengde & 1.16 & 1.41 & 0.21 & 0.63 & 1.22 \\
\hline
\end{tabular}

26

\begin{tabular}{|c|c|c|c|c|c|c|}
\hline Period & Property & $\mathbf{T}_{-24}$ & $\mathbf{T}_{-18}$ & $T_{-12}$ & $\mathbf{T}_{-6}$ & $\mathbf{T}_{\mathbf{0}}$ \\
\hline \multirow{4}{*}{ Ep1 } & $\mathrm{R}_{\mathrm{BC}}$ & 3.6 & 4.0 & 5.2 & 7.8 & 8.7 \\
\hline & GMD & 97 & 115 & 128 & 139 & 134 \\
\hline & $\mathrm{N}$ & 28994 & 15494 & 15204 & 15592 & 19242 \\
\hline & $\mathrm{Cr}$ & 40 & 93 & 75 & 7 & 34 \\
\hline \multirow{4}{*}{ Ep2 } & $\mathrm{R}_{\mathrm{BC}}$ & 2.1 & 3.6 & 2.3 & 5.7 & 3.8 \\
\hline & GMD & 91 & 104 & 102 & 119 & 106 \\
\hline & $\mathrm{N}$ & 23909 & 15189 & 17961 & 10994 & 20121 \\
\hline & $\mathrm{Cr}$ & 1.2 & 0.14 & 0.01 & 95 & 13 \\
\hline \multirow{4}{*}{ Ep3 } & $\mathrm{R}_{\mathrm{BC}}$ & 6.9 & 13.2 & 3.2 & 4.1 & 7.6 \\
\hline & GMD & 13 & 74 & 96 & 95 & 126 \\
\hline & $\mathrm{N}$ & 22234 & 11880 & 13481 & 14241 & 12945 \\
\hline & $\mathrm{Cr}$ & 59 & 81.4 & 6.2 & 8.8 & 1 \\
\hline \multirow{4}{*}{ Ep4 } & $\mathrm{R}_{\mathrm{BC}}$ & 2.3 & 6.2 & 3.6 & 5.4 & 6.9 \\
\hline & GMD & 102 & 98 & 95 & 111 & 117 \\
\hline & $\mathrm{N}$ & 19754 & 12805 & 21116 & 10536 & 17199 \\
\hline & $\mathrm{Cr}$ & 98 & 56 & 68 & 25 & 1 \\
\hline \multirow{4}{*}{ Ep5 } & $\mathrm{R}_{\mathrm{BC}}$ & 69 & 10.0 & 2.9 & 2.1 & 6.6 \\
\hline & GMD & 29 & 114 & 99 & 95 & 124 \\
\hline & $\mathrm{N}$ & 8617 & 8086 & 16494 & 28211 & 13696 \\
\hline & $\mathrm{Cr}$ & 100 & 100 & 50 & 4 & 78 \\
\hline \multirow{4}{*}{ Ep6 } & $\mathrm{R}_{\mathrm{BC}}$ & 1.8 & 2.4 & 4.6 & 5.9 & 4.6 \\
\hline & GMD & 98 & 103 & 111 & 129 & 116 \\
\hline & $\mathrm{N}$ & 31691 & 23691 & 17885 & 12897 & 21955 \\
\hline & $\mathrm{Cr}$ & 54 & 0.17 & 0.01 & 65 & 19 \\
\hline
\end{tabular}

Table S2. Aerosol properties along transport, including geometric mean diameter (GMD [nm]), mass ratio of coating to $\mathrm{BC}\left(\mathrm{R}_{\mathrm{BC}}\right)$, number concentration $(\mathrm{N})$ and contribution of region source to $\mathrm{BC}(\mathrm{Cr}[\%]) . \mathrm{T}_{0}$ means ending points of back trajectories and $T_{n}$ means $n$ hours before arriving at the ending point. 